\title{
INVESTIGACIONES
}

\section{Concepciones alternativas mayoritarias sobre Universo en profesores de Física en formación*}

\author{
Most common alternative conceptions about Universe in future Physics teachers
}

\author{
Leonor Huerta Cancino \\ Departamento de Física, Facultad de Ciencia, Universidad de Santiago de Chile \\ Telf.: (56) 227181288. Correo electrónico: leonor.huerta@usach.cl
}

\begin{abstract}
RESUMEN
Este artículo presenta un avance de los resultados y principales conclusiones de un estudio que buscó identificar y analizar las concepciones alternativas mayoritarias (CAM) de estudiantes de Pedagogía en Física, en contenidos seleccionados sobre Universo, para tres cohortes (2014, 2015 y 2016). Para identificar las CAM se utilizó una versión modificada de la prueba estandarizada Astronomy Diagnostic Test v2.0. Se definieron categorías de análisis que permitieron seleccionar un conjunto de CAM para las cuales se determinó la necesidad de desarrollar secuencias didácticas específicas para facilitar a los futuros profesores de Física el aprendizaje del conocimiento científico vigente sobre esos contenidos. Los resultados muestran un conjunto acotado de CAM, similares para las tres cohortes, en los tópicos: movimientos en la esfera celeste, tamaños y distancias a escala, fases de la Luna, eclipses y estaciones del año, y propiedades de las estrellas.
\end{abstract}

Palabras clave: ideas previas, ciencias de la Tierra y el Universo, formación inicial docente.

\begin{abstract}
This paper presents a preview of results and key findings, of a study that seeks to identify and to analyze most common alternative conceptions (CAM), of future Physics teachers, in selected contents about Universe, for three cohort (2014, 2015 and 2016). Categories of analysis were defined allowing to select a set of CAM for which it was determined the need to develop specific teaching sequences to facilitate future Physics teachers learning the current scientific knowledge on those contents. To identify CAM, it was used a modified version of the standardized test Astronomy Diagnostic Test v2.0. Results show a limited set of CAM, similar to the three groups, on the following topics: movements in the celestial sphere, sizes and distances at scale, moon phases, eclipses and seasons, and star's properties.
\end{abstract}

Key words: misconceptions, Earth and Universe sciences, teacher education. 


\section{INTRODUCCIÓN}

\subsection{CONCEPCIONES ALTERNATIVAS}

Los y las estudiantes poseen concepciones sobre diversos fenómenos naturales, temas y situaciones, que no necesariamente son acordes con los conceptos científicos vigentes (CCV). En diversas investigaciones realizadas sobre el conocimiento o ideas que tienen los alumnos sobre el mundo socio-natural, realizadas durante las décadas de los 70 y los 80, Cubero (1994) consigna una serie de términos usados que no presentan una definición explícita por parte de los investigadores, quienes incluso los usan como sinónimos. Entre los términos encontrados por Cubero (1994) se encuentran: Concepciones erróneas (misconceptions), Preconcepciones (preconceptions), Ciencia de los niños (children's science), Marcos alternativos (alternative frameworks), Concepciones alternativas (alternative conceptions), Razonamiento espontáneo (spontaneous reasoning), Ideas ingenuas (naive ideas), Ideas pre-instruccionales (pre-instructional ideas), Representaciones (representations), Esquemas conceptuales alternativos. Cubero considera que el término concepciones falsas o erróneas (misconceptions) tiene claramente una connotación negativa, al considerar erróneo todo conocimiento (ya sean creencias, ideas, modelos, teorías o marcos conceptuales) que no se corresponda con el conocimiento científico vigente. Otra manera de subvalorar las ideas de los alumnos es calificarlas de preconceptos: es decir, no alcanzarían la categoría de conceptos como sí lo es el conocimiento académico o científico. En esta misma línea, Cubero considera que los términos marcos o concepciones alternativas, esquemas o ideas previas no involucran tal sub-valoración de las ideas de los alumnos, ya que estas son valoradas como conocimiento cotidiano: los alumnos han desarrollado concepciones que deben ser conciliadas con el conocimiento científico vigente. Es así como en el contexto de esta investigación, se utilizará el término concepciones alternativas, entendiéndolas como representaciones mentales de las personas sobre el mundo natural que difieren del conocimiento científico vigente (son "alternativas" justamente porque difieren de los conceptos científicos).

Cuellar (2009) propone mejorar la enseñanza-aprendizaje de las Ciencias Naturales en general, y de la Física en particular, partiendo por identificar las concepciones alternativas de los estudiantes sobre una temática particular, y analizar cómo estas concepciones alternativas influyen en el proceso de aprendizaje, facilitándolo o limitándolo, según la relación que se establezca entre las concepciones alternativas del alumno y los conceptos científicos que presente el profesor. Esto es relevante, ya que diversos estudios han mostrado que una característica importante de las concepciones alternativas es su persistencia (Varela et al., 2013). Después de años de instrucción escolar, los alumnos mantienen sus concepciones alternativas: son persistentes, es decir, no se modifican fácilmente por medio de la enseñanza tradicional de la ciencia, incluso cuanto la instrucción es reiterada (Mora \& Herrera, 2009); es decir, los alumnos no se deshacen totalmente de sus concepciones alternativas: lo que hacen es agregarles significados (Moreira \& Greca, 2003).

Además, puede ocurrir que las concepciones alternativas del propio docente influyan en que sus estudiantes formen concepciones alternativas que no sean coherentes con el CCV (Kikas, 2004; Schoon, 1995). Para prevenir que esto ocurra, se hace necesario que los profesores sean capaces de diferenciar entre los conceptos científicos y las concepciones alternativas más comunes en los tópicos que enseñan en la escuela (Kikas, 2004). Sin embargo, estudios en diversos países señalan que las dificultades que tienen los docentes 
para enseñar ciencias se debe principalmente a su falta de confianza y un nivel insuficiente de dominio conceptual (Kriner, 2004). En este sentido, un paso importante consiste en identificar las CAM durante la formación de profesores de ciencias para, a continuación, elaborar diseños didácticos que faciliten el aprendizaje de los conceptos científicos, con el objetivo de formar profesores que no utilicen concepciones alternativas al momento de enseñar contenidos científicos.

\subsection{CIENCIAS DE LA TIERRA Y EL UNIVERSO}

En el caso particular de las Ciencias de la Tierra y el Universo (Earth and Space Sciences, en inglés), temas como fases de la Luna, estaciones del año, eclipses, entre otros, tienen como característica común que son fenómenos de ocurrencia cotidiana y periódica, y para los cuales los alumnos desarrollan concepciones alternativas bien estructuradas y persistentes. Por ejemplo, investigaciones sobre concepciones alternativas respecto a estaciones del año, muestran que los alumnos piensan que estas se producen debido a la mayor o menor distancia de la Tierra al Sol (Varela et al., 2013): así, cuando la Tierra está más cerca del Sol se está en verano y cuando está más lejos, en invierno. Esto es coherente con la experiencia cotidiana de situarse a menor o mayor distancia de una estufa. También es coherente con la imagen exagerada de la órbita elíptica de la Tierra alrededor del Sol, con una gran excentricidad (cuando en realidad la excentricidad es muy pequeña), lo que se traduce en que los alumnos interpreten que en un momento dado la Tierra está mucho más lejos del Sol que en otras partes de su órbita.

Por otra parte, los contenidos abordados por las CTU son interdisciplinares: consideran elementos de Física, Química, Geología y Biología, lo que representa una demanda de docentes competentes en articular una gran variedad de conocimientos interdisciplinares.

Otro factor a considerar es que las Ciencias de la Tierra y el Universo (CTU) han sido incorporadas desde hace relativamente poco tiempo como contenido de los programas de estudio de diversos países, por lo que muchos profesores de física no enseñaron estos contenidos de manera regular en décadas anteriores. En el caso chileno, desde el año 1990 hasta el año 2009, los contenidos sobre CTU en enseñanza media se concentraban en una única unidad de $2^{\circ}$ medio (Unidad 3: La Tierra y su Entorno). Sin embargo, estudios de cobertura curricular realizados por el Ministerio de Educación (MINEDUC) a partir del año 2000, mostraron que casi el 80\% de los profesores de Física encuestados no enseñaban los contenidos de la unidad La Tierra y su Entorno (MINEDUC, 2007). Un paso en pos de mejorar esta situación consistió en establecer ejes de conocimiento, que abarcan desde $1^{\circ}$ básico hasta $4^{\circ}$ medio, en el ajuste curricular del año 2009. Uno de estos ejes corresponde a Tierra y Universo (Unidad de Currículum y Evaluación, 2009), lo que significó un gran desafío para los profesores en ejercicio, ya que no enseñaban estos contenidos.

Estos cambios curriculares suponen a su vez un desafío para las carreras de Pedagogía en Física, que deben preparar a los futuros profesores en estos nuevos contenidos. El MINEDUC, además, establece explícitamente que los conocimientos mínimos e imprescindibles que cada profesor o profesora debe saber en el ámbito de su disciplina y de la enseñanza de la misma, así como las competencias genéricas, disposiciones y actitudes profesionales necesarias para desempeñarse eficazmente (MINEDUC, 2012) a través de los Estándares Orientadores para Carreras de Pedagogía en Educación Media.

Los estándares se organizan en dos categorías: (i) Estándares Disciplinarios para la Enseñanza, que definen las competencias específicas para enseñar la disciplina, y 
(ii) Estándares Pedagógicos, que reúnen las competencias necesarias para el adecuado desarrollo del proceso de enseñanza. Respecto a Física, los estándares incluyen nueve áreas, una de las cuales corresponde a Tierra y Universo (el estándar 9):

Describe y comprende los aspectos principales asociados a la formación y evolución de cuerpos y estructuras cósmicas, así como la estructura y dinámica de la Tierra. El futuro profesor o profesora comprende que los fenómenos astronómicos y aquellos relacionados con la dinámica terrestre han cobrado fuerza en los últimos años, tanto por su importancia como por el avance que han tenido las técnicas y los instrumentos asociados a su estudio. Por ello, el futuro profesor o profesora comprende los conceptos, leyes, modelos y teorías que dan cuenta de los principales fenómenos asociados a la formación, evolución, dinámica y características de la Tierra y de grandes estructuras del Universo. Es capaz de cuantificar y establecer relaciones entre los tamaños de los cuerpos y estructuras celestes, así como las distancias entre ellos. Conoce y utiliza analogías, modelos, problemas y estrategias desafiantes que permitan construir y evidenciar aprendizajes, habilidades, contenidos y actitudes relacionadas con la formación y evolución de cuerpos y estructuras cósmicas, así como la estructura y dinámica de la Tierra. (MINEDUC, 2012, p. 203).

En otras palabras, el propio MINEDUC describe explícitamente las competencias disciplinares en el área de Tierra y Universo que deben poseer los egresados de carreras de Pedagogía en Física, y en este contexto también desde las exigencias institucionales es que se hace evidente la importancia de conocer las concepciones alternativas sobre estos contenidos durante la formación inicial docente de profesores de Física. A partir de esta información se pueden diseñar e implementar secuencias didácticas que faciliten a los futuros profesores incorporar nuevos significados a sus esquemas mentales, y que les permitan articular explicaciones sobre estos contenidos utilizando concepciones científicas vigentes.

\subsection{OBJETIVOS}

El objetivo principal de esta investigación fue identificar y analizar las concepciones alternativas mayoritarias de estudiantes de la Pedagogía en Física y Matemática de la Universidad de Santiago de Chile (USACH), en contenidos seleccionados del área de las Ciencias de la Tierra y el Universo del Currículum Nacional vigente en Chile (MINEDUC, 2009, 2013), y en relación a los Estándares Orientadores para las carreras de Pedagogía en Educación Media (MINEDUC, 2012).

Este objetivo se enmarca dentro del trabajo de tesis de la autora para optar al grado de doctor en el área de la Física Educativa. En este trabajo se seleccionan parte de las CAM identificadas en el presente estudio para desarrollar secuencias didácticas específicas en la FID de profesores de Física.

\section{METODOLOGÍA}

La investigación se plantea como un estudio del tipo descriptivo comparativo, de cohortes, en un diseño mixto que involucra elementos cualitativos y cuantitativos en el análisis de las evidencias. 
Participantes: los sujetos de estudio fueron estudiantes inscritos en la asignatura semestral Física del Universo, de segundo año de la Pedagogía en Física y Matemática de la USACH, en los años 2014, 2015 y 2016. El muestreo es no probabilístico, del tipo por conveniencia: esto es, estudiantes inscritos en un mismo curso (MacMillan \& Schumacher, 2005). Este estudio contó con la participación de tres cohortes:

- $\quad$ grupo G-2014 con 41 alumnos (16 mujeres y 25 hombres).

- $\quad$ grupo G-2015 con 34 alumnos (17 mujeres y 17 hombres).

- $\quad$ grupo G-2016 con 27 alumnos (9 mujeres y 18 hombres).

Instrumento: el diseño de la investigación contempló usar como instrumento de recogida de datos una prueba de conceptos estandarizada, para identificar concepciones alternativas de los estudiantes sobre contenidos relativos al Universo. Para seleccionar una prueba estandarizada que sirviera a los objetivos de esta investigación, se revisó la literatura especializada y se verificó que los conceptos abordados por las preguntas estuvieran en concordancia con los contenidos descritos en los Programas de Estudios del Currículum Nacional vigente en el país (Bases Curriculares y Marco Curricular ajustado). Se seleccionó el Astronomy Diagnostic Test $^{1}$ (ADT versión 2.0), desarrollado por el grupo multi-institucional Collaboration for Astronomy Education Research (CAER) en el año 1999 (Hufnagel et al., 2000; Zeilik, 2003). Es una prueba escrita que consta de 33 preguntas de selección múltiple de las cuales las primeras 21 tratan sobre conceptos de astronomía comúnmente presentes en cursos introductorios de astronomía en carreras de pregrado universitarias (Hufnagel, 2002), es decir, el ADT está diseñado para ser utilizado en estudiantes universitarios (en sus primeros niveles de pregrado) que estén tomando un primer curso de astronomía, como es el caso de los sujetos de estudio. Las últimas 12 preguntas son de tipo demográfico (edad, género, origen étnico, entre otros).

En el ADT v.2.0, para cada una de las 21 preguntas sobre astronomía, se presentan entre tres y cinco alternativas de las cuales solamente una expresa el conocimiento científico vigente (alternativa CCV) y el resto expresa alguna concepción alternativa reportada en investigaciones previas en el área. Conocer el porcentaje de selección de estas alternativas que expresan concepciones alternativas permitirá determinar la de mayor selección o alternativa CAM (Gangui, 2007).

El test ADT v2.0 cuenta con una versión oficial en español. Sin embargo, se realizaron algunas modificaciones que fueron mínimas y no alteraron de manera alguna el objetivo del test. La primera modificación consistió en expresar las unidades de distancia en el sistema métrico (preguntas 3 y 15) porque es el sistema operante en el lugar de residencia de los sujetos de estudio. La segunda modificación afectó a la pregunta $\mathrm{n}^{\mathrm{o}} 12$, en la cual se cambió la referencia a la constelación de la Osa Mayor (que no es posible observar desde el lugar de residencia de los sujetos de estudio), por la constelación de Orión (que sí es posible observar). 


\section{EVIDENCIAS}

Durante agosto de 2014 se aplicó la versión 2.0 del ADT en español (Hufnagel et al., 2000), con las modificaciones descritas incorporadas, al grupo G-2014. En marzo de 2015, la misma prueba fue aplicada al grupo G-2015, y en junio de 2016 esta se aplicó al grupo G-2016. Los resultados para cada una de las 21 preguntas de contenido, para los tres grupos, se presentan en la Tabla 1: el porcentaje de alumnos que seleccionaron la alternativa correcta (\% CCV); el porcentaje de alumnos que seleccionaron la alternativa incorrecta de mayor nivel de elección por parte de los participantes (\% CAM); y la letra (a, $\mathrm{b}, \mathrm{c}, \ldots)$ que identifica la alternativa correspondiente a la alternativa CAM.

Tabla 1. Resumen de resultados por pregunta

\begin{tabular}{|c|c|c|c|c|c|c|c|c|c|}
\hline & \multicolumn{4}{|c|}{ G-2014 } & \multicolumn{4}{c|}{ G-2015 } & \multicolumn{3}{c|}{ G-2016 } \\
\hline Pregunta & $\begin{array}{c}\% \\
\text { CCV }\end{array}$ & $\begin{array}{c}\% \\
\text { CAM }\end{array}$ & $\begin{array}{c}\text { Alternativa } \\
\text { CAM }\end{array}$ & $\begin{array}{c}\% \\
\text { CCV }\end{array}$ & $\begin{array}{c}\% \\
\text { CAM }\end{array}$ & $\begin{array}{c}\text { Alternativa } \\
\text { CAM }\end{array}$ & $\begin{array}{c}\% \\
\text { CCV }\end{array}$ & $\begin{array}{c}\% \\
\text { CAM }\end{array}$ & $\begin{array}{c}\text { Alternativa } \\
\text { CAM }\end{array}$ \\
\hline 1 & 29,3 & 31,7 & a & 17,6 & 55,9 & a & 31,3 & 56,3 & a \\
\hline 2 & 19,5 & 34,1 & a & 5,9 & 41,2 & a & 6,3 & 43,8 & a \\
\hline 3 & 22,0 & 22,0 & c & 5,9 & 32,4 & c & 37,5 & 43,8 & c \\
\hline 4 & 87,8 & 9,8 & a & 82,4 & 17,6 & a & 68,8 & 31,3 & a \\
\hline 5 & 22,0 & 70,7 & a & 17,6 & 76,5 & a & 6,3 & 93,8 & a \\
\hline 6 & 4,9 & 26,8 & a & 5,9 & 32,4 & a & 0,0 & 31,3 & a \\
\hline 7 & 22,0 & 39,0 & a & 0,0 & 64,7 & a & 25,0 & 31,3 & b \\
\hline 8 & 53,7 & 29,3 & b & 29,4 & 44,1 & b & 50,0 & 18,8 & b \\
\hline 9 & 31,7 & 31,7 & c & 41,2 & 26,5 & b & 43,8 & 37,5 & c \\
\hline 10 & 29,3 & 34,1 & d & 20,6 & 17,6 & a & 12,5 & 31,3 & b \\
\hline 11 & 63,4 & 14,6 & b & 50,0 & 17,6 & b & 62,5 & 18,8 & c \\
\hline 12 & 56,1 & 7,3 & c y e & 35,3 & 20,6 & e & 37,5 & 31,3 & c y d \\
\hline 13 & 73,2 & 9,8 & e & 47,1 & 26,5 & d & 56,3 & 18,8 & d \\
\hline 14 & 39,0 & 29,3 & e & 26,5 & 47,1 & e & 12,5 & 43,8 & e \\
\hline 15 & 34,1 & 39,0 & b & 17,6 & 44,1 & b & 18,8 & 56,3 & b \\
\hline 16 & 61,0 & 14,6 & d & 58,8 & 11,8 & d & 43,8 & 25,0 & c \\
\hline 17 & 26,8 & 29,3 & c & 20,6 & 32,4 & c & 18,8 & 37,5 & d \\
\hline 18 & 41,5 & 19,5 & a & 32,4 & 23,5 & a & 31,3 & 31,3 & a \\
\hline 19 & 34,1 & 22,0 & b & 50,0 & 14,7 & b & 43,8 & 43,8 & b \\
\hline 20 & 53,7 & 22,0 & c & 38,2 & 26,5 & e & 56,3 & 31,3 & c \\
\hline 21 & 56,1 & 24,4 & a & 41,2 & 38,2 & a & 43,8 & 37,5 & a \\
\hline
\end{tabular}


Los resultados presentados en la Tabla 1, permiten identificar la alternativa CAM para cada una de las 21 preguntas de contenido junto a su porcentaje de selección por parte de los alumnos de cada grupo. Cabe destacar que los grupos G-2014 y G-2015 presentan exactamente la misma CAM en 17 de las 21 preguntas del test y, de hecho, en 12 preguntas la CAM es la misma en los tres grupos. En la Tabla 2, se presentan los enunciados de las 21 preguntas del test y las CAM identificadas:

Tabla 2. Identificación de las Concepciones. Alternativas Mayoritarias

\begin{tabular}{|c|c|c|c|c|}
\hline $\mathrm{N}^{\mathrm{o}}$ & Enunciado de la Pregunta & $\begin{array}{l}\text { Alternativa } \\
\text { Correcta } \\
(\mathrm{CCV})\end{array}$ & $\begin{array}{l}\text { Alternativa } \\
\text { CAM } \\
\text { G-2014 } \\
\text { y G-2015 }\end{array}$ & $\begin{array}{l}\text { Alternativa } \\
\text { CAM } \\
\text { G-2016 }\end{array}$ \\
\hline 1 & $\begin{array}{l}\text { ¿Observando desde la ciudad en que } \\
\text { vives, cuándo es que un asta de bandera } \\
\text { vertical no produce sombra alguna de- } \\
\text { bido a que el Sol se encuentra directa- } \\
\text { mente sobre el asta bandera? }\end{array}$ & $\begin{array}{l}\text { Nunca desde } \\
\text { este lugar }\end{array}$ & $\begin{array}{l}\text { Todos los días } \\
\text { al mediodía }\end{array}$ & $\begin{array}{l}\text { Todos los días al } \\
\text { mediodía }\end{array}$ \\
\hline 2 & $\begin{array}{l}\text { ¿En qué fase se debe de encontrar la } \\
\text { Luna cuando aparenta cubrir por com- } \\
\text { pleto al Sol (un eclipse)? }\end{array}$ & Nueva & Llena & Llena \\
\hline 3 & $\begin{array}{l}\text { Imagina que estás construyendo un mo- } \\
\text { delo a escala de la Tierra y la Luna. Vas } \\
\text { a utilizar una pelota de basketball de } 30 \\
\mathrm{~cm} \text { de diámetro para representar a la } \\
\text { Tierra y una pelota de tenis de } 7 \mathrm{~cm} \\
\text { para la Luna. ¿Qué tan lejos de la super- } \\
\text { ficie de la pelota de basketball debes } \\
\text { colocar la pelota de tenis para mantener } \\
\text { la escala de distancia apropiada? }\end{array}$ & $9 \mathrm{~m}$ & $90 \mathrm{~cm}$ & $90 \mathrm{~cm}$ \\
\hline 4 & $\begin{array}{l}\text { Tienes dos pelotas del mismo tamaño y } \\
\text { aspereza, y puedes ignorar la resisten- } \\
\text { cia del aire. Una de ellas es pesada y la } \\
\text { otra es mucho más ligera. Sostienes una } \\
\text { en cada mano a la misma altura sobre el } \\
\text { suelo. Las dejas caer al mismo tiempo. } \\
\text { ¿Qué pasará? }\end{array}$ & $\begin{array}{l}\text { Ambas tocarán } \\
\text { el suelo al } \\
\text { mismo tiempo }\end{array}$ & $\begin{array}{l}\text { La más pesada } \\
\text { tocará el suelo } \\
\text { primero }\end{array}$ & $\begin{array}{l}\text { La más pesada } \\
\text { tocará el suelo } \\
\text { primero }\end{array}$ \\
\hline 5 & $\begin{array}{l}\text { ¿Cómo se compara la velocidad de las } \\
\text { ondas de radio con la velocidad de la } \\
\text { luz visible? }\end{array}$ & $\begin{array}{c}\text { Ambas viajan a } \\
\text { la misma } \\
\text { velocidad }\end{array}$ & $\begin{array}{l}\text { Las ondas de } \\
\text { radio son } \\
\text { mucho más } \\
\text { lentas }\end{array}$ & $\begin{array}{l}\text { Las ondas de } \\
\text { radio son mucho } \\
\text { más lentas }\end{array}$ \\
\hline 6 & $\begin{array}{l}\text { Los astronautas dentro del Transborda- } \\
\text { dor Espacial flotan en la cabina al orbi- } \\
\text { tar la Tierra porque... }\end{array}$ & $\begin{array}{l}\text { Están cayendo } \\
\text { de la misma } \\
\text { manera que el } \\
\text { Transbordador } \\
\text { Espacial }\end{array}$ & $\begin{array}{l}\text { No hay } \\
\text { gravedad en el } \\
\text { espacio }\end{array}$ & $\begin{array}{l}\text { No hay gravedad } \\
\text { en el espacio }\end{array}$ \\
\hline
\end{tabular}




\begin{tabular}{|c|c|c|c|c|}
\hline 7 & $\begin{array}{l}\text { Imagina que la órbita de la Tierra fuera } \\
\text { cambiada a ser un círculo perfecto alre- } \\
\text { dedor del Sol tal que la distancia al Sol } \\
\text { nunca cambiara. ¿Cómo afectaría esto a } \\
\text { las Estaciones del Año? }\end{array}$ & $\begin{array}{l}\text { Continuaría- } \\
\text { mos experi- } \\
\text { mentando las } \\
\text { Estaciones de } \\
\text { la misma ma- } \\
\text { nera que ahora }\end{array}$ & $\begin{array}{c}\text { Ya no experi- } \\
\text { mentaríamos } \\
\text { diferencias entre } \\
\text { las Estaciones } \\
\text { del Año }\end{array}$ & $\begin{array}{l}\text { Continuaríamos } \\
\text { experimentando } \\
\text { las Estaciones, } \\
\text { pero la diferen- } \\
\text { cia entre ellas } \\
\text { sería mucho } \\
\text { MENOS notoria }\end{array}$ \\
\hline 8 & ¿De dónde proviene la energía del Sol? & $\begin{array}{l}\text { De la combina- } \\
\text { ción de ele- } \\
\text { mentos ligeros } \\
\text { en otros más } \\
\text { pesados }\end{array}$ & \begin{tabular}{|c|} 
De la \\
descomposición \\
de elementos \\
pesados en otros \\
más ligeros.
\end{tabular} & $\begin{array}{l}\text { De la } \\
\text { descomposición } \\
\text { de elementos } \\
\text { pesados en otros } \\
\text { más ligeros. }\end{array}$ \\
\hline 9 & $\begin{array}{l}\text { El } 22 \text { de septiembre, aproximadamente, } \\
\text { el Sol se oculta directamente hacia el } \\
\text { Oeste como lo muestra el diagrama de } \\
\text { abajo. ¿Dónde aparentaría ocultarse } \\
\text { dos semanas después? }\end{array}$ & $\begin{array}{l}\text { Más hacia el } \\
\text { Sur }\end{array}$ & $\begin{array}{l}\text { [Más hacia el } \\
\text { Norte] } \\
\text { (G-2014) } \\
\text { [En el mismo } \\
\text { lugar] } \\
\text { (G-2015) }\end{array}$ & $\begin{array}{l}\text { Más hacia el } \\
\text { Norte }\end{array}$ \\
\hline 10 & $\begin{array}{l}\text { Así es como se vería el cielo al medio- } \\
\text { día en cierta fecha si pudiéramos ver las } \\
\text { estrellas durante el día. El Sol se en- } \\
\text { cuentra cerca de las estrellas de la cons- } \\
\text { telación de Géminis. ¿Cerca de cuál } \\
\text { constelación esperarías localizar al Sol } \\
\text { al atardecer? }\end{array}$ & Géminis & $\begin{array}{l}\text { Leo (G-2014) } \\
\text { Tauro (G-2015) }\end{array}$ & Cáncer \\
\hline 11 & $\begin{array}{l}\text { ¿Qué tan lejos de la Tierra se encuentra } \\
\text { el Transbordador Espacial (cuando está } \\
\text { en el espacio) comparado con la } \\
\text { distancia de la Luna? }\end{array}$ & $\begin{array}{c}\text { Muy cerca de } \\
\text { la Tierra }\end{array}$ & $\begin{array}{l}\text { Aproximada- } \\
\text { mente a medio } \\
\text { camino de la } \\
\text { Luna }\end{array}$ & $\begin{array}{l}\text { Muy cerca de la } \\
\text { Luna }\end{array}$ \\
\hline 12 & $\begin{array}{l}\text { Vistas desde tu ciudad, las estrellas de } \\
\text { la constelación de Orión pueden ser co- } \\
\text { nectadas con líneas imaginarias para } \\
\text { formar la figura del cazador: dos trape- } \\
\text { zoides conectados por el cinturón de } \\
\text { Orión (las "tres Marías"). ¿Hasta dónde } \\
\text { tendríamos que viajar para observar un } \\
\text { cambio notable en la figura de la cons- } \\
\text { telación? }\end{array}$ & $\begin{array}{l}\text { A una estrella } \\
\text { distante }\end{array}$ & $\begin{array}{c}\text { [Europa] y } \\
\text { [Plutón] } \\
\text { (G-2014) } \\
\text { Plutón (G-2015) }\end{array}$ & $\begin{array}{c}\text { [Europa] y [la } \\
\text { Luna] }\end{array}$ \\
\hline 13 & $\begin{array}{l}\text { ¿Cuál de estas listas está arreglada } \\
\text { correctamente en orden de lo más } \\
\text { cercano a lo más lejano de la Tierra? }\end{array}$ & $\begin{array}{l}\text { Luna, Sol, } \\
\text { Plutón, } \\
\text { estrellas }\end{array}$ & $\begin{array}{l}\text { [Luna, Plutón, } \\
\text { Sol, estrellas] } \\
\text { (G-2014) } \\
\text { [Luna, Sol, } \\
\text { estrellas, } \\
\text { Plutón] } \\
\text { (G-2015) }\end{array}$ & $\begin{array}{l}\text { Luna, Sol, } \\
\text { estrellas, Plutón }\end{array}$ \\
\hline
\end{tabular}




\begin{tabular}{|c|c|c|c|c|}
\hline 14 & $\begin{array}{l}\text { ¿Cuál de estas cosas te harían pesar la } \\
\text { mitad de lo que ahora pesas? }\end{array}$ & $\begin{array}{l}\text { Quitar la mitad } \\
\text { de la masa de } \\
\text { la Tierra }\end{array}$ & $\begin{array}{l}\text { Más de una de } \\
\text { las respuestas } \\
\text { anteriores son } \\
\text { correctas }\end{array}$ & $\begin{array}{l}\text { Más de una de } \\
\text { las respuestas } \\
\text { anteriores son } \\
\text { correctas }\end{array}$ \\
\hline 15 & $\begin{array}{l}\text { Una persona está leyendo un periódico } \\
\text { parada a una distancia de } 1,5 \mathrm{~m} \text { de una } \\
\text { mesa sobre la cual tenemos un foco } \\
\text { encendido de } 100 \text { watts. Imagina que la } \\
\text { mesa se retira a una distancia de } 3 \mathrm{~m} \text {. } \\
\text { ¿Cuántos focos en total tendrían que ser } \\
\text { colocados sobre la mesa para alumbrar } \\
\text { al periódico con la misma cantidad de } \\
\text { luz que antes? }\end{array}$ & Cuatro focos & Dos focos & Dos focos \\
\hline 16 & $\begin{array}{l}\text { ¿Qué se puede decir sobre la } \\
\text { localización del centro del Universo } \\
\text { según las teorías y observaciones } \\
\text { modernas? }\end{array}$ & $\begin{array}{l}\text { Que el } \\
\text { Universo no } \\
\text { tiene centro }\end{array}$ & $\begin{array}{l}\text { Que una galaxia } \\
\text { desconocida y } \\
\text { lejana se } \\
\text { encuentra en el } \\
\text { centro }\end{array}$ & $\begin{array}{l}\text { Que nuestra } \\
\text { galaxia, la Vía } \\
\text { Láctea, se } \\
\text { encuentra en el } \\
\text { centro }\end{array}$ \\
\hline 17 & $\begin{array}{l}\text { ¿De qué color son las estrellas de mayor } \\
\text { temperatura? }\end{array}$ & Azul & Rojo & Blanco \\
\hline 18 & $\begin{array}{l}\text { El siguiente diagrama muestra a la } \\
\text { Tierra, al Sol y a } 5 \text { posibles posiciones } \\
\text { diferentes para la Luna. ¿Qué posición } \\
\text { de la Luna causaría que desde la Tierra } \\
\text { se la observarse como en la figura de la } \\
\text { derecha? }\end{array}$ & $\begin{array}{l}\mathrm{D} \text { (nueva } \\
\text { visible) }\end{array}$ & A (llena) & A (llena) \\
\hline 19 & $\begin{array}{l}\text { Observas a la Luna Llena salir por el } \\
\text { Este. ¿Cómo se vería } 6 \text { horas después? }\end{array}$ & C (llena) & B (creciente) & B (creciente) \\
\hline 20 & $\begin{array}{l}\text { Tu dedo pulgar es apenas suficiente } \\
\text { para cubrir por completo al Sol cuando } \\
\text { extiendes el brazo completamente. } \\
\text { ¿Cuál objeto utilizarías para cubrir al } \\
\text { Sol si estuvieras en Saturno, el cual se } \\
\text { encuentra } 10 \text { veces más alejado del Sol } \\
\text { que la Tierra? }\end{array}$ & $\begin{array}{l}\text { Un fideo de } \\
\text { espagueti. }\end{array}$ & $\begin{array}{l}\text { [Un lápiz] } \\
\text { (G-2014) } \\
\text { [Un pelo] } \\
\text { (G-2015) }\end{array}$ & Un lápiz \\
\hline 21 & $\begin{array}{l}\text { El Calentamiento Global se cree que es } \\
\text { causado por }\end{array}$ & $\begin{array}{l}\text { La cantidad } \\
\text { creciente de } \\
\text { dióxido de } \\
\text { carbono en la } \\
\text { atmósfera }\end{array}$ & $\begin{array}{l}\text { la destrucción } \\
\text { de la capa de } \\
\text { ozono }\end{array}$ & $\begin{array}{l}\text { La destrucción } \\
\text { de la capa de } \\
\text { ozono }\end{array}$ \\
\hline
\end{tabular}

Fuente: elaboración propia. 


\section{ANÁLISIS DE LAS CAM}

Para realizar una comparación entre las alternativas CCV y CAM para cada una de las 21 preguntas del test, parte de la información de la Tabla 1 se presenta de manera gráfica, mostrando el porcentaje de selección de la alternativa correcta (CCV, barra azul) junto a la alternativa incorrecta con mayor porcentaje de selección (CAM, barra roja) para los grupos G-2014 (Gráfico 1), G-2015 (Gráfico 2), y G-2016 (Gráfico 3).

Gráfico 1. Porcentajes obtenidos por la alternativa CCV (en azul) y por la alternativa CAM (en rojo) para el grupo G-2014

\section{Porcentaje de Selección de Alternativas CCV - CAM (G-2014)}

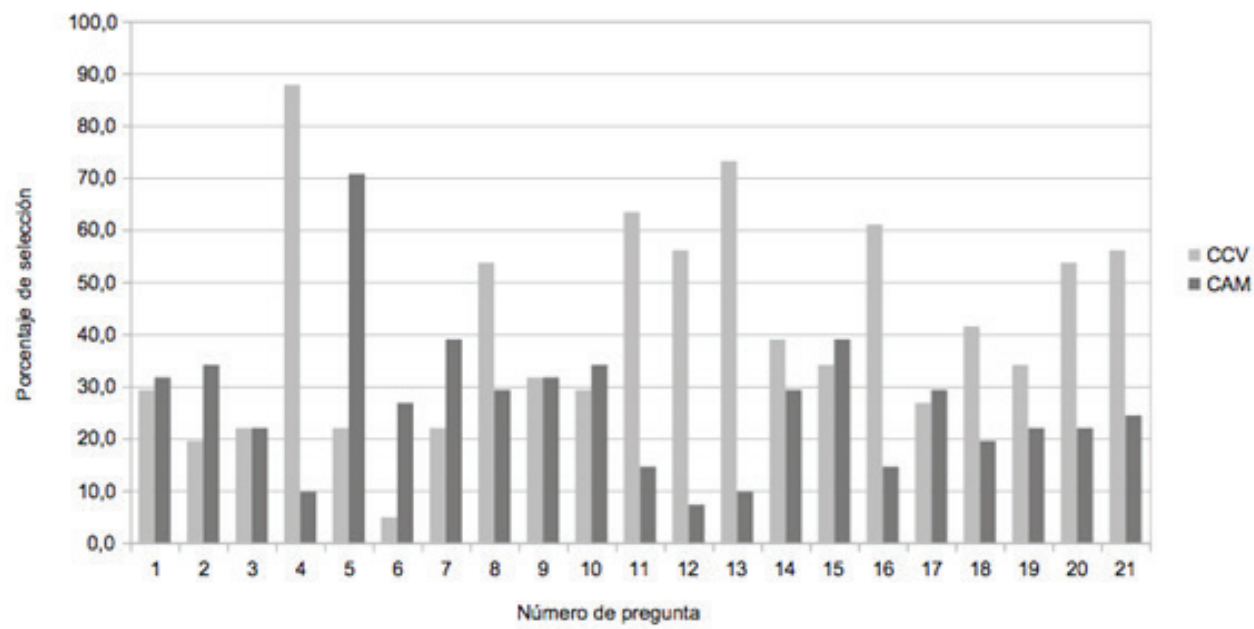

Fuente: elaboración propia. 
Gráfico 2. Porcentajes obtenidos por la alternativa CCV (en azul) y por la alternativa CAM (en rojo) para el grupo G-2015

\section{Porcentaje de Selección de Alternativas CCV - CAM (G-2015)}

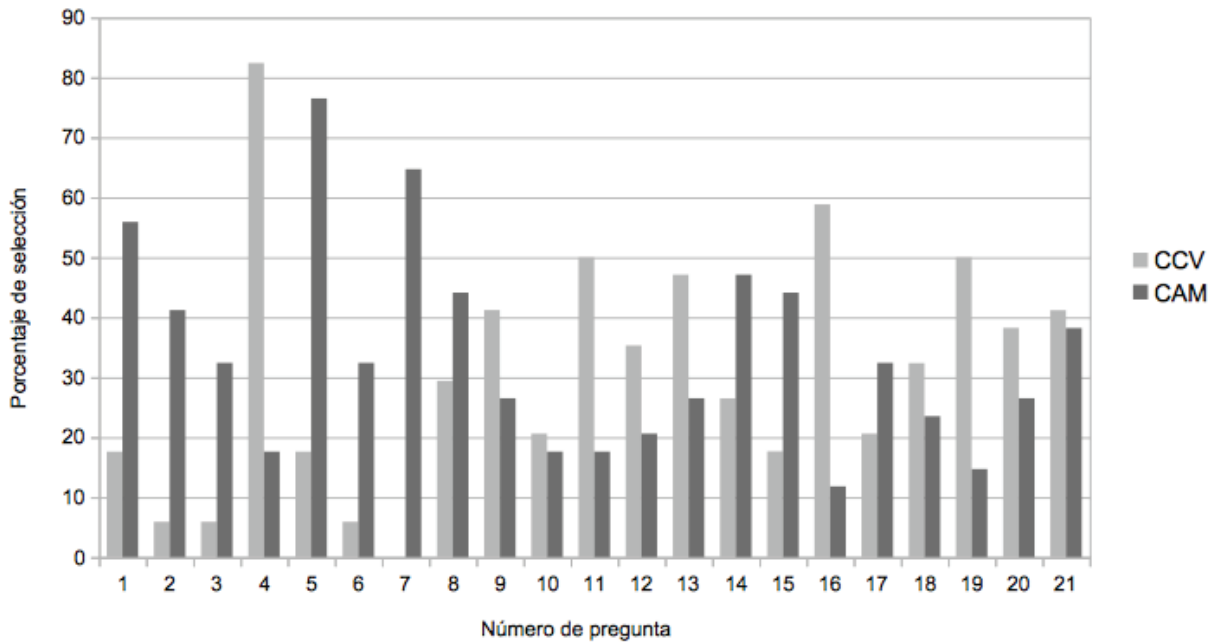

Fuente: elaboración propia.

Gráfico 3. Porcentajes obtenidos por la alternativa CCV (en azul) y por la alternativa CAM (en rojo) para el grupo G-2016

\section{Porcentaje de Selección de Alternativas CCV - CAM (G-2016)}

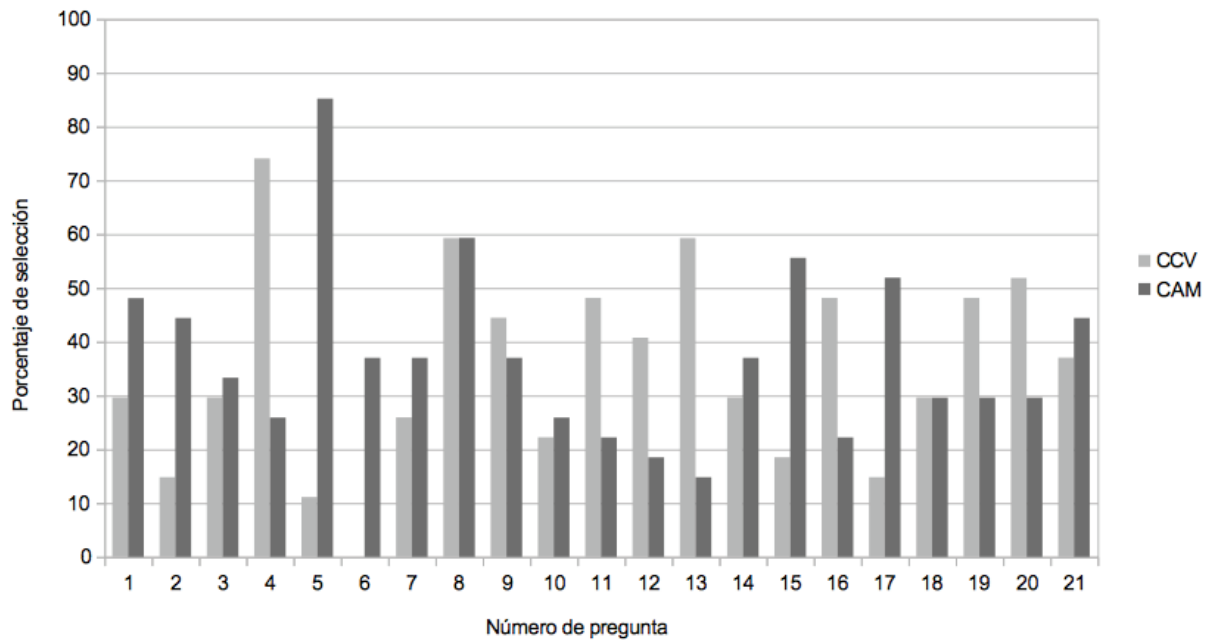

Fuente: elaboración propia. 
En los gráficos se observan preguntas para las cuales la alternativa CAM presenta un porcentaje de selección similar al de la alternativa CCV y otras para las cuales los porcentajes de selección son significativamente diferentes. Para analizar estos resultados, se definieron tres categorías de análisis:

- $\quad \mathrm{C}_{1}$ : preguntas en las cuales la alternativa CCV y la alternativa CAM presentan porcentajes de selección similares (diferencias menores un 5\%): \%CAM $\approx \% \mathrm{CCV}$.

- $\quad \mathrm{C}_{2}$ : preguntas en que la alternativa CAM tiene significativamente un mayor porcentaje de selección que la alternativa $\mathrm{CCV}$ (diferencias mayores a un 5\%): $\% \mathrm{CAM}>\% \mathrm{CCV}$.

- $\quad \mathrm{C}_{3}$ : preguntas para las cuales la alternativa CCV tiene significativamente un mayor porcentaje de selección que la alternativa CAM (diferencias mayores a un 5\%): $\% \mathrm{CAM}<\% \mathrm{CCV}$.

En la categoría $\mathrm{C}_{3}$ está el tipo de preguntas que lograron el mejor resultado, ya que la alternativa CCV fue elegida en mayor porcentaje que la alternativa CAM por parte de los alumnos. En otras palabras, para las preguntas de esta categoría, los alumnos identificaron la respuesta correcta (en coherencia con el conocimiento científico vigente) por sobre las respuestas que expresaban concepciones alternativas.

En cambio, las categorías $\mathrm{C}_{1}$ y $\mathrm{C}_{2}$ agrupan preguntas sobre conceptos para los cuales los alumnos identifican, en un porcentaje importante, respuestas que no coinciden con el conocimiento científico vigente. En las preguntas de estas dos categorías, la alternativa CAM iguala o supera el porcentaje en que fue seleccionada la alternativa correcta.

La Tabla 3 muestra los números de las preguntas clasificadas en las categorías $\mathrm{C}_{1}, \mathrm{C}_{2}$ $\mathrm{y} \mathrm{C}_{3}$, destacando con asterisco simple (*) aquellas en las cuales la alternativa CAM obtuvo puntajes iguales o por sobre el $50 \%$ de elección, y con doble asterisco (**) aquellas en las cuales la alternativa CCV obtuvo puntajes iguales o por sobre el $50 \%$ de elección por parte de los alumnos.

Tabla 3. Preguntas por categoría

\begin{tabular}{|c|c|c|c|}
\hline Categoría & G-2014 & G-2015 & G-2016 \\
\hline $\mathrm{C}_{1}$ & $1,3,9,10,15,17$ & 10,21 & $3,10,18$ \\
\hline $\mathrm{C}_{2}$ & $2,5^{*}, 6,7$ & $\begin{array}{c}1^{*}, 2,3,5^{*}, 6,7^{*}, 8 \\
14,15,17\end{array}$ & $\begin{array}{c}1,2,5^{*}, 6,7,8^{*}, 14, \\
15^{*}, 17^{*}, 21\end{array}$ \\
\hline $\mathrm{C}_{3}$ & $\begin{array}{c}4 * *, 8^{* *}, 11^{* *}, 12 * * \\
13^{* *}, 14,16^{* *}, 18 \\
19,20^{* *}, 21^{* *}\end{array}$ & $\begin{array}{c}4 * *, 9,11^{* *}, 12,13 \\
16^{* *}, 18,19^{* *}, 20\end{array}$ & $\begin{array}{c}4 * *, 9,11,12,13^{* *} \\
16,19,20 * *\end{array}$ \\
\hline
\end{tabular}


Los datos en la Tabla 3 muestran que la categoría $\mathrm{C}_{3}$, que corresponde a preguntas para las cuales la alternativa CCV tiene significativamente un mayor porcentaje de elección que la alternativa CAM, por una parte concentra el mayor número de preguntas, como también el mayor número de preguntas cuya alternativa CCV fue seleccionada en porcentajes iguales o superiores al 50\%. Es decir, los alumnos mostraron un mejor rendimiento justamente en estas preguntas, lo que para las tres cohortes esto ocurre en las preguntas $n^{\circ} 4,11,12,13$, 16,19 y 20.

En conjunto, la totalidad de las preguntas en las categorías $\mathrm{C}_{1}$ y $\mathrm{C}_{2}$ abordan conceptos para los cuales los alumnos manifiestan una concepción alternativa mayoritaria que compite fuertemente con la concepción científica vigente. En la categoría $\mathrm{C}_{2}$ hay cuatro preguntas que coinciden en los tres grupos: las $\mathrm{n}^{\mathrm{o}}$ 2, 5, 6 y 7. En las preguntas 1, 5, 7, 8, 15 y 17, los alumnos seleccionaron la alternativa CAM con porcentajes superiores al 50\%, por lo que deben ser analizadas más profundamente.

\section{REFLEXIONES Y CONSIDERACIONES FINALES}

Los resultados permiten establecer, en primer término, que los alumnos en las tres cohortes eligieron en gran medida las mismas alternativas con concepciones alternativas, es decir, presentan en gran medida el mismo conjunto de CAM. Este resultado es importante en el sentido de que la variedad de CAM identificadas queda limitada a un conjunto reducido: se debe recordar que el test incluye en promedio cuatro concepciones alternativas para cada una de las 21 preguntas, lo que se traduce en 84 concepciones alternativas seleccionables por los participantes del estudio. Cada cohorte pudo aportar con un mínimo de 21 CAM (totalizando 63 CAM entre los tres grupos). Sin embargo, los resultados muestran un conjunto no mayor a 30 CAM considerando las tres cohortes.

En segundo término, el análisis de las categorías definidas establece que siete preguntas de la categoría $\mathrm{C}_{3}$ corresponden a contenidos para los cuales los alumnos de las tres cohortes mostraron un buen nivel al seleccionar la alternativa CCV (correcta) en porcentajes superiores a la alternativa CAM (incorrecta mayoritaria). Estas preguntas corresponden a las $\mathrm{n}^{\mathrm{o}}$ 4,11,12,13,16, 19 y 20, las cuales además fueron seleccionadas por más del 50\% de los alumnos. Para estos contenidos, no se hace imprescindible elaborar secuencias didácticas específicas, justamente, porque los alumnos de las tres cohortes mostraron un buen nivel reconociendo como respuesta correcta la alternativa que expresaba el conocimiento científico vigente.

Respecto a las categorías $\mathrm{C}_{1}$ y $\mathrm{C}_{2}$, estas reúnen todas las preguntas del test para las cuales la alternativa CCV obtuvo niveles menores de selección, en contraste con los altos porcentajes en que fueron seleccionadas las alternativas CAM. Son estas preguntas $\left(\mathrm{n}^{\mathrm{o}} 1\right.$, $2,3,5,6,7,8,910,14,15,17,18$ y 21) las que tratan sobre contenidos para los cuales los alumnos identifican como respuesta correcta una alternativa CAM en porcentajes incluso superiores al 50\%. Los contenidos abordados en estas preguntas se pueden ordenar en tres niveles:

- Conceptos Básicos: Movimientos en la esfera celeste, tamaños y distancias a escala.

- Conceptos Intermedios: Fases de la Luna, Eclipses y Estaciones del año.

- Conceptos Avanzados: Temperatura, color y brillo de las estrellas. 
A modo de ejemplo, para la pregunta $\mathrm{n}^{\mathrm{o}} 2$ los alumnos identifican mayoritariamente que la Luna debe estar en la fase Llena cuando se produce un eclipse de Sol, cuando en realidad debe estar en fase Nueva. Esto se puede interpretar de varias maneras, como por ejemplo: (i) los alumnos no identifican claramente las características de la fase de Luna Nueva, (ii) los alumnos relacionan la fase de Luna Llena con un mayor tamaño de la Luna, necesario para poder "tapar" al Sol durante un eclipse, (iii) los alumnos no conocen las características de un eclipse de Sol, entre otras posibles interpretaciones.

Para la pregunta $n^{\circ} 7$, los alumnos consideran que la distancia entre la Tierra y el Sol juega un papel preponderante como causa de las estaciones del año. En la pregunta 17, los alumnos de dos de los tres grupos relacionan el color rojo con mayor temperatura de las estrellas, probablemente, porque en su experiencia cotidiana las llaves para las cañerías de agua caliente se identifican con el color rojo y las de agua fría con el color azul, aun cuando la experiencia cotidiana también debería reportarles la observación de llamas más azules o más rojizas (o anaranjadas) para el fuego de la cocina o el calefón.

En la Tabla 4 se encuentra un resumen de las CAM seleccionadas a partir del análisis de las categorías definidas:

Tabla 4. Descripción de las Concepciones Alternativas Mayoritarias

\begin{tabular}{|c|l|}
\hline $\mathrm{N}^{\mathrm{o}}$ & \multicolumn{1}{|c|}{ Descripción de la CAM } \\
\hline 1 & $\begin{array}{l}\text { Noción equivocada de la eclíptica: piensan que el Sol pasa por el cénit todos los días a } \\
\text { mediodía en Santiago de Chile. }\end{array}$ \\
\hline 2 & Identifican la Luna llena como la fase en la que se pueden producir eclipses de Sol. \\
\hline 3 & No conocen la proporción tamaño-distancia para el sistema Tierra-Luna. \\
\hline 5 & Piensan que las ondas de radio son mucho más lentas que la luz. \\
\hline 6 & $\begin{array}{l}\text { Piensan que no hay gravedad o que la gravedad disminuye notablemente fuera de la } \\
\text { atmósfera terrestre y por eso un astronauta flota en un transbordador espacial que } \\
\text { orbita la Tierra. }\end{array}$ \\
\hline 7 & Piensan que las estaciones del año se originan por la distancia Tierra-Sol. \\
\hline 8 & $\begin{array}{l}\text { Piensan que el Sol genera energía principalmente por procesos de fisión nuclear en su } \\
\text { interior. }\end{array}$ \\
\hline 9 & $\begin{array}{l}\text { No relacionan las estaciones del año con los cambios en la posición aparente del Sol } \\
\text { (visto desde la Tierra). }\end{array}$ \\
\hline 10 & $\begin{array}{l}\text { Piensan que el Sol cambia su posición relativa a las constelaciones durante un mismo } \\
\text { día. }\end{array}$ \\
\hline 14 & $\begin{array}{l}\text { No relacionan apropiadamente las variables involucradas en la interacción gravitacional } \\
\text { entre una persona y la Tierra. }\end{array}$ \\
\hline 15 & $\begin{array}{l}\text { No conocen el decaimiento del brillo con el cuadrado de la distancia (creen que la } \\
\text { relación es inversamente proporcional). }\end{array}$ \\
\hline 17 & $\begin{array}{l}\text { Piensan que las estrellas rojas o blancas tienen mayor temperatura superficial que las } \\
\text { azules (no asocian frecuencia de una OEM con la energía). }\end{array}$ \\
\hline 18 & $\begin{array}{l}\text { No identifican correctamente las fases de la Luna en función de la posición relativa } \\
\text { Tierra-Luna-Sol. }\end{array}$ \\
\hline 21 & Identifican como causa del calentamiento global la reducción de la capa de ozono. \\
\hline
\end{tabular}


Como reflexión final, una vez identificadas y analizadas las concepciones alternativas mayoritarias de los profesores de Física en formación, se hace necesario diseñar e implementar secuencias didácticas específicas, que les faciliten el aprendizaje de estos contenidos científicos ${ }^{2}$. Lograr que los futuros profesores de Física enseñen a sus propios alumnos contenidos acordes con el conocimiento científico vigente en lugar de sus concepciones alternativas, significará un aporte en pos de mejorar la calidad de la educación y en la alfabetización científica de la comunidad. En cuanto a los profesores ya en ejercicio, sería importante que el sistema les ofrezca cursos de apropiación curricular sobre estos contenidos, en el marco de programas de Educación Continua.

\section{REFERENCIAS BIBLIOGRÁFICAS}

Cubero, R. (1994). Concepciones alternativas, preconceptos, errores conceptuales... ¿distinta terminología y un mismo significado? Investigación en la Escuela, 23, 33-42.

Cuellar, Z. (2009). Las concepciones alternativas de los estudiantes sobre la naturaleza de la materia. Revista Iberoamericana de Educación, 50(2), 1-10.

Gangui, A. (2007). Los científicos y la alfabetización en astronomía. Anales AFA, 18(1), 24-27.

Hufnagel, B. (2002). Development of the Astronomy Diagnostic Test. Astronomy Education Review, l(1), 47-51.

Hufnagel, B., Slater, T., Deming, G., Adams, J., Lindell Adrian, R., Brick, C., \& Zeilik, M. (2000). Pre-Course Results from the Astronomy Diagnostic Test. Publications of the Astronomical Society of Australia, 17(2), 152-155.

Kikas, E. (2004). Teachers' conceptions and misconceptions concerning three natural phenomena. Journal of Research in Science Teaching, 41(5), 432-448.

Kriner, A. (2004). Las fases de la Luna, ¿cómo y cuándo enseñarlas? Ciência \& Educação, 10(1), 111-120.

MacMillan, J., \& Schumacher, S. (2005). Investigación Educativa: Una introducción conceptual. Madrid: Pearson Educación.

MINEDUC. (2007). Información Consolidada sobre cobertura curricular en segundo ciclo básico y medio. Santiago: Autor. Recuperado desde http://www.educarchile.cl/UserFiles/P0001/File/ Consolidado\%20Cobertura\%20Curricular\%20en\%20Enseanza\%20Media5.doc

MINEDUC. (2009). Objetivos Fundamentales y Contenidos Mínimos Obligatorios de la Educación Básica y Media. Actualización 2009. Santiago: Autor. Recuperado desde http://www. curriculumenlineamineduc.cl/605/articles-34641_bases.pdf

MINEDUC. (2012). Estándares orientadores para las carreras de Pedagogía en Educación Media. Santiago: Autor. Recuperado desde http://portales.mineduc.cl/usuarios/cpeip/File/ librosestandaresvale/libromediafinal.pdf

MINEDUC. (2013). Bases Curriculares $7^{\circ}$ básico a $2^{\circ}$ medio. Santiago: Autor. Recuperado desde http://educrea.cl/wp-content/uploads/2015/04/Bases-Curriculares- $7^{\circ}$-Básico-a-2º-Medio.pdf

Mora, C., \& Herrera, D. (2009). Una revisión sobre ideas previas del concepto de fuerza. Revista Latinoamericana de Física Educativa, 3(1), 72-86.

Moreira, M., \& Greca, I. (2003). Cambio conceptual: análisis crítico y propuestas a la luz de la teoria del aprendizaje significativo. Ciência e Educação, 9(2), 301-315.

Schoon, K. J. (1995). The origin and extent of alternative conceptions in the earth and space sciences:

Como parte del trabajo de tesis doctoral de la autora, se diseñaron e implementaron secuencias didácticas para los contenidos Estaciones del Año, Fases de la Luna y Eclipses, en el marco de la asignatura Física del Universo del programa de FID para Profesores de Física de la Universidad de Santiago de Chile. 
A survey of preservice elementary teachers. Journal of Elementaty Science Education, 7(2), 27-46.

Unidad de Currículum y Evaluación. (2009). Ajuste Curricular. Principales énfasis y modificaciones curriculares para continuar fortaleciendo el aprendizaje de los alumnos y alumnas del país. Santiago: Ministerio de Educación. Recuperado desde http://ww2.educarchile.cl/UserFiles/ P0001/File/ajuste_curricular/Articulo_Fundamentos_Ajuste_Ciencias_Naturales_300309.pdf

Varela, M., Pérez, U., Serrallé, J., \& Arias, A. (2013). Evolución de las concepciones sobre astronomía del profesorado en formación tras una intervención educativa con actividad desde simulación. IX Congreso internacional sobre investigación en didáctica de las ciencias. Girona, España.

Zeilik, M. (2003). Birth of the Astronomy Diagnostic Test: Prototest Evolution. Astronomy Education Review, 1(2), 46-52. 